\title{
The persistence and performance of phosphate-solubilizing Gluconacetobacter liquefaciens qzr14 in a cucumber soil
}

\author{
Jingjing Wang ${ }^{1}$ Huan Wang ${ }^{1,2} \cdot{\text { Tingting } \mathrm{Yin}^{1,2} \cdot \text { Song } \mathrm{Xu}^{1} \cdot \text { Wei Zhao }}^{1} \cdot$ \\ Jin Wang ${ }^{1}$ - Zhiyong Huang ${ }^{1}$
}

Received: 12 October 2016/Accepted: 22 August 2017/Published online: 30 August 2017

(C) Springer-Verlag GmbH Germany 2017

\begin{abstract}
The persistence and performance of plant growth-promoting microorganisms (PGPMs) in soil are considered critical features for effectiveness, yet they are poorly understood. Here, we investigated the colonization and activity of a new PGPM, phosphate-solubilizing Gluconacetobacter liquefaciens qzr14, in a pot culture experiment using cucumber as test crop for 20 days. The number of G. liquefaciens and bacterial diversity in the rhizosphere and bulk soil were monitored by real-time PCR and DGGE, respectively. Soil phosphorus and cucumber biomass were also examined. G. liquefaciens qzr14 effectively colonized the rhizosphere soil (bacterial density ranging from $2.70 \times 10^{8}$ to $1.18 \times 10^{9}$ copies per gram dry soil). $G$. liquefaciens qzr14 inoculation had significantly positive effects on bacterial diversity (BD) of the rhizosphere and bulk soil and the ratio of soluble phosphorus to total phosphorus (SP/TP). The number of G. liquefaciens in the rhizosphere soil was significantly related to SP/TP and the $\mathrm{BD}$ of the rhizosphere and bulk soil. BD in rhizosphere soil was significantly related to SP/TP and BD in bulk soil. Based on the results of correlation analysis, we inferred that the introduced $G$. liquefaciens qzr14 effectively
\end{abstract}

Electronic supplementary material The online version of this article (doi:10.1007/s13205-017-0926-z) contains supplementary material, which is available to authorized users.

$\triangle$ Zhiyong Huang

huang_zy@tib.cas.cn

1 Tianjin Key Laboratory for Industrial Biological Systems and Bioprocessing Engineering, Tianjin Institute of Industrial Biotechnology, Chinese Academy of Sciences, No. 32, West 7th Road, Tianjin Airport Economic Area, Tianjin 300308, People's Republic of China

2 College of Biology Engineering, Tianjin University of Science and Technology, Tianjin 300457, China colonized the rhizosphere of cucumber, and then expanded its bacterial community by solubilizing soil phosphorus. The expanded bacterial communities might promote cucumber growth by some new functions.

Keywords Bacterial community - Colonization · Gluconacetobacter liquefaciens · Phosphate-solubilizing activity $\cdot$ Plant growth-promoting microorganisms (PGPMs)

\section{Introduction}

The application of plant growth-promoting microorganisms (PGPMs) has been considered as a highly attractive agricultural method, because it substantially reduces the use of chemical fertilizers and pesticides (Baris et al. 2014; Kumar et al. 2009; Sathya et al. 2016). PGPMs promote plant growth by (i) supplying nutrients (such as nitrogenfixing bacteria and phosphate-solubilizing bacteria); (ii) producing plant hormones (such as Bradyrhizobium spp. and Rhizobium spp.); (iii) controlling or inhibiting the activity of plant pathogens (such as Bacillus spp. and Pseudomonas spp.); (iv) improving soil structure; and (v) inducing systemic resistance (Bhattacharyya and Jha 2012; Bloemberg and Lugtenberg 2001). Apart from these features, PGPMs also promote plant growth by modifying the structure and function of microbial communities (Trabelsi and Mhamdi 2013). Recently, an increasing number of PGPMs being commercialized for various crops worldwide (Owen et al. 2015). Several applications of PGPMs into soils had been successful; i.e., these have resulted in the colonization of soil and plant roots to a level sufficiently high for the intended purpose (Bhattacharjee et al. 2012; Chabot et al. 1996; Luna et al. 2012). However, 
various failures or inconsistencies in achieving the objective have also been reported (Holmberg et al. 2012; Vanelsas et al. 1986; Von Felten et al. 2010). For example, Burkholderia tropica increased tomato fruit yields by $17 \%$ in the first year, but only $6 \%$ in the second year (Bernabeu et al. 2015). Some ACC-utilizing or $\mathrm{N}_{2}$-fixing bacteria effectively promoted the growth of plants in a sterilized environment, whereas its plant growth-promoting effects were weaker in a non-sterilized environment (Liu et al. 2014). These inconsistencies and failures have raised concerns about the perspective of the great practical potential offered by microbial releases into soils. A key factor involved in the lack of success was the rapid decline of the size of the populations of inoculants to levels ineffective to achieve the objective following its introduction into soil. Population declines have been observed in a wide variety of newly introduced bacteria in rhizosphere soil, including Pseudomonads fluorescent (Von Felten et al. 2010), Bradyrhizobium sp. (Dudeja and Khurana 1989), Azospirillum sp. (Bashan et al. 1995), and Rhizobium spp.(Postma et al. 1990). It has been suggested that the scarcity of available nutrient sources to soil microbes and the hostility of the soil environment to incoming microbes due to a myriad of adverse abiotic and biotic factors might be reasons for the decline in PGPM populations in rhizosphere soil (vanVeen et al. 1997). Another factor involved in the lack of success may be related to the activity of PGPMs in soil. Some reports showed that the plant growthpromoting activities can be affected by fungicides, substrate, temperature, $\mathrm{pH}$, microorganism, and so on (Liu et al. 2014).

Previously, our group isolated new PGPM bacteria, phosphate-solubilizing bacterial qzr14, from the rhizosphere soil of eggplant (Yin et al. 2015). Pot experiments showed that qzr14 inoculation can significantly increase the height (27.56\%), dry (98.46\%), and fresh $(57.01 \%)$ weight of cucumber seedlings. Here, we investigated the colonization and activity of the qzr14 in soil for 20 days. This study may facilitate in further understanding of the colonization and activity of PGPMs in soil.

\section{Materials and methods}

\section{Microorganisms}

The bacterial strain qzr14 was isolated from the rhizosphere soil of eggplant in Anshan, Liaoning, China (N $40^{\circ} 59^{\prime} 59.41^{\prime \prime}$, E $123^{\circ} 0^{\prime} 29.22^{\prime \prime}$ ) in a previous study (Yin et al. 2015). The pot experiment showed that strain qzr14 can significantly increase the height $(27.56 \%)$ as well as dry $(98.46 \%)$ and fresh $(57.01 \%)$ weight of cucumber seedlings (Yin et al. 2015). 16S rDNA sequence analysis using primers $27 \mathrm{~F} / 1492 \mathrm{R}$ was performed to identify strain qzr14 (Tanner et al. 1999). Specific primers of Gluconacetobacter diazotrophicus, G. sacchari, and G. liquefaciens were used to verify the species of strain qzr14 (Franke-Whittle et al. 2005). Growth in the presence of $0.01 \%$ malachite green was tested (Franke et al. 1999). Nitrogen fixation, phosphate solubilization and potassium solubilization capacity of strain qzr14 were assessed using selective mediums (Jiménez et al. 2011; Nautiyal 1999). P-solubilization was further determined using the Mo-blue method (Chen et al. 2006). Indoleacetic acid (IAA) and siderophore production were measured using Salkowski reagents and CAS medium, respectively (Rana et al. 2011).

\section{Pot experiments}

Soil was collected from the 0 to $20 \mathrm{~cm}$ depth of a cropped field in Anshan, Liaoning, China (N 40 59'59.41", E $\left.123^{\circ} 0^{\prime} 29.22^{\prime \prime}\right)$. The soil had a $\mathrm{pH}$ of 6.8 , organic matter content of $23.5 \mathrm{~g} \mathrm{~kg}^{-1}$, total nitrogen content of $14.2 \mathrm{~g} \mathrm{~kg}^{-1}$, total phosphate content of $107 \mathrm{mg} \mathrm{kg}^{-1}$, total potassium content of $31 \mathrm{~g} \mathrm{~kg}^{-1}$, available nitrogen content of $147 \mathrm{mg} \mathrm{kg}^{-1}$, available phosphate content of $6 \mathrm{mg} \mathrm{kg}^{-1}$, and available potassium content of $376 \mathrm{mg} \mathrm{kg}^{-1}$. The soil was ground and passed through a $1-\mathrm{mm}$ sieve prior to use in the pot experiment. The plant species used in the present study was Cucumis sativus L. (Chinese Academy of Agricultural Science, Beijing, China). The experiment was conducted in a plant growth chamber (RXZ, Ningbo Jiangnan Instrument Plant, Ningbo, China) at the Tianjin Institute of Industrial Biotechnology, Chinese Academy of Science. Plastic cylinder pots with a diameter of $8 \mathrm{~cm}$ and a height of $9 \mathrm{~cm}$ were used. Each pot contained a uniform mix of $118.9 \mathrm{~g}$ dry soil and $1.1 \mathrm{~g} \mathrm{Ca}_{3}(\mathrm{PO} 4)_{2}$. Cucumber seeds (No. 8 Zhongnong, China) were soaked in sterilized distillationdistillation $\mathrm{H}_{2} \mathrm{O}$ for $10 \mathrm{~min}$. The seeds were then placed on nutritive soil for 7 days to allow germination. Seven-dayold seedlings were removed from the nutritive soil and transplanted into pots (1 seedling per pot). The bacterial strain qzr14 was cultivated overnight in beef extract peptone broth containing $5 \%$ glucose at $30{ }^{\circ} \mathrm{C}$. The cultures were centrifuged at $3000 \mathrm{rpm}$ for $5 \mathrm{~min}$. The resulting pellets were resuspended in a $0.9 \% \mathrm{NaCl}$ solution and diluted to a density of about $1 \times 10^{8}$ cells $\mathrm{mL}^{-1}$. The experimental setup included two treatments: inoculated bacterial strain qzr14 (Q) and a control check (CK). Approximately $20 \mathrm{~mL}$ of bacterial suspension (Q) or $0.9 \%$ $\mathrm{NaCl}$ solution (CK) was applied to the seedlings after transplantation. Fifty plants were grown for each treatment. The plants were grown for 20 days in the plant growth chamber under controlled environmental conditions of 14-h days at $28{ }^{\circ} \mathrm{C}$ and 10 -h nights at $12{ }^{\circ} \mathrm{C}$. Irrigation was 
manually performed every second day. Every 4 days, three plants of each treatment were harvested. Rhizosphere and bulk soil were collected and stored at $4{ }^{\circ} \mathrm{C}$ (for soil characterization) or $-80{ }^{\circ} \mathrm{C}$ (for soil DNA isolation) until analysis.

\section{DNA extraction}

DNA was extracted from $0.25 \mathrm{~g}$ of frozen rhizosphere or bulk soil using a PowerSoil ${ }^{\circledR}$ DNA isolation kit (Mo Bio Laboratories, CA, USA). The quantity and purity of DNA were determined using a NanoDrop (NanoDrop-1000, Thermo Scientific, USA). The DNA was then stored at $-20{ }^{\circ} \mathrm{C}$ before use.

\section{Real-time PCR assay}

Quantification of copy number of strain qzr14 was performed using a real-time PCR assay. Real-time PCR experiments were conducted in a 7500 Fast Real-Time PCR System (Applied Biosystem, Foster City, CA, USA). Strain qzr14-specific primers (GLF 5'-GGCTGCATTT GATACGTCCA-3'; GLR 5'-GCGTTAACTACGACA CTGAATGA-3') were used (Franke-Whittle et al. 2005). Each PCR was performed in a total reaction volume of 20 $\mu \mathrm{L}$, which consisted of using $10 \mu \mathrm{L}$ of SYBR Select Master Mix $(2 \times)$ (Applied Biosystem, Foster City, CA, USA), $250 \mathrm{nM}$ of each primer, $1 \mu \mathrm{L}$ of the template DNA ( $\sim 3 \mathrm{ng} \mu \mathrm{L}^{-1}, A_{260 / 280} \sim 1.9, A_{260 / 230} \sim 2.0$ ), and $7 \mu \mathrm{L}$ of $\mathrm{ddH}_{2} \mathrm{O}$. The final two-step cycling program included a 2-min initial pre-incubation at $95^{\circ} \mathrm{C}$ followed by 40 cycles of $95{ }^{\circ} \mathrm{C}$ for $15 \mathrm{~s}$ and $60{ }^{\circ} \mathrm{C}$ for $1 \mathrm{~min}$.

Standards for real-time PCR assays were prepared as described elsewhere (Wang et al. 2014). Briefly, the specific $16 S$ rRNA gene of strain qzr14 was PCR-amplified from extracted DNA with the primers GLF/GLR, and the PCR products were cloned into a pMD19-T Simple Vector (TaKaRa, Dalian, China). Plasmids used as standards for quantitative analyses were extracted from the correct insert clones of each target gene using a Plasmid Miniprep Kit (BIOMIGA, Shanghai, China). The concentration of plasmid DNA was determined on a NanoDrop (NanoDrop1000, Thermo Scientific, USA), and the copy numbers of the target genes were calculated directly from the concentration of the extracted plasmid DNA. Ten-fold serial dilutions of each known copy number of the plasmid DNA were subjected to a real-time PCR assay in triplicate to generate an external standard curve.

\section{Denaturing gradient gel electrophoresis (DGGE)}

Denaturing gradient gel electrophoresis analysis of bacterial community of the rhizosphere and bulk soil was performed with the DCode Universal Mutation Detection System (Bio-Rad Laboratories, Hercules, USA) and primers 341F GC-518R. Similar amounts of the PCR products were loaded onto polyacrylamide gradient gels with a denaturing gradient of $40-70 \%$ (100\% denaturant containing $7 \mathrm{M}$ urea and $40 \%$ formamide). Electrophoreses were run at $180 \mathrm{~V}$ for $6 \mathrm{~h}$. The gels were analyzed using the software Quantity One (Bio-Rad Laboratories) (Wang et al. 2014). UPGMA algorithms were used to cluster the DGGE patterns. The bands in the DGGE gel were excised and amplified with the primers $341 \mathrm{~F} / 518 \mathrm{R}$. The purified PCR products were ligated into the pMD19-T Simple Vector (TaKaRa, Dalian, China). The resulting ligation mix was transformed into Escherichia coli DH5a competent cells following the instructions of the manufacturer. The positive clones were amplified using the above primers with GC clamp, and then verified by DGGE. The correct clone was selected for sequencing. The sequences were aligned with BLAST, and a phylogenetic tree was constructed using the neighbor-joining method provided in MEGA version 5.0 with a bootstrap value of 1000 replicates. The Shannon diversity index $H$ was used to calculate the diversity of bacterial communities.

\section{Soil phosphorus}

The bulk soil in each pot was mixed and sampled. All soil samples were air-dried and sieved through a $150-\mu \mathrm{m}$ mesh sieve before storing for analysis. Soil total phosphate was determined using a Soil Total Phosphate Kit (COMIN, Suzhou Comin Biotechnology Co., Ltd, China). Solubilized phosphorus was determined using the molybdenum blue method (Xie et al. 2013).

\section{Fresh weight of cucumber seedlings}

Every 4 days, cucumber seedlings of three pots were harvested. Soils adhering to the rhizosphere were washed with $\mathrm{ddH}_{2} \mathrm{O}$. After air-drying, the fresh weight of the cucumber seedlings was measured on an analytical balance.

\section{Statistical analysis}

The copy numbers of strain qrz14 quantified by real-time PCR assay were log-transformed and normalized to per gram dry soil prior to statistical analysis. All statistical analyses were performed with SPSS version 17.0. Mean comparisons were performed between inoculation treatment $(\mathrm{Q})$ and control check (CK) using an independent $t$ test at individual time points. Pearson correlation analysis was adopted to analyze the correlations among G. liquefaciens populations, bacterial diversity, soil phosphorous, 
and fresh weight of cucumber seedlings. $P<0.05$ was considered statistically significant.

\section{Results and discussion}

\section{Properties of bacterial strain qzr14}

16S rDNA sequence analysis of bacterial strain qzr14 (Accession no. KP715459) showed high similarity with G. liquefaciens (99\%), G. sacchari (99\%), and G. diazotrophicus (98\%). Among genus Gluconacetobacter, only G. liquefaciens and G. sacchari showed growth on malachite green medium (Franke et al. 1999). Strain qzr14 can grow on $0.01 \%$ malachite green medium (Fig. S1). PCR analysis showed that strain qzr14 only can be amplified by the primer of G. liquefaciens (Fig. S2). These assays validated that strain qzr14 was G. liquefaciens. G. diazotrophicus has been widely applied in sugarcane fields based on its nitrogen-fixing ability (Munoz-Rojas and Caballero-Mellado 2003; Saravanan et al. 2008). However, the plant growth-promoting activity of G. liquefaciens has never been reported except our previous work. Chemical assays showed that strain qzr14 could grow on Ashby medium, P-solubilization medium, and K-solubilization medium, and that strain qzr14 had IAA secreting ability, and could not form orange holes on CAS medium. The P-solubilization capacity of strain qzr14 was $270 \mathrm{mg} \mathrm{L}^{-1}$ (Table S1).

\section{Persistence of G. liquefaciens qzr14 in the bulk and rhizosphere soil of cucumber}

The colonization and persistence of introduced PGPM in soil are the bases to exert their plant growth-promoting abilities (Compant et al. 2010). The colonization and persistence of introduced PGPM in rhizosphere soil had been widely investigated (Bashan et al. 1995; Couillerot et al. 2010; Pellegrino et al. 2012; Troxler et al. 2012). Population declines have been observed in a wide variety of newly introduced bacteria in rhizosphere soil (Vanelsas et al. 1986; Von Felten et al. 2010). However, our results showed that in the rhizosphere soil, the number of G. liquefaciens was significantly higher in the inoculation treatment $[\mathrm{Q}(\mathrm{R})]$ (bacterial density ranging from $2.70 \times 10^{8}$ to $1.18 \times 10^{9}$ copies per gram dry soil) than that in the control check $[\mathrm{CK}(\mathrm{R})]$ (bacterial density ranging from $8.89 \times 10^{7}$ to $1.92 \times 10^{8}$ copies per gram dry soil) at 4-20 days after inoculation $(P<0.001)$ (Fig. 1). The number of $G$. liquefaciens significantly increased over time in both $\mathrm{Q}(\mathrm{R})$ and $\mathrm{CK}(\mathrm{R})$. The number of $G$. liquefaciens showed a four-fold increase in $\mathrm{Q}(\mathrm{R})$, but only a two-fold enhancement in $\mathrm{CK}(\mathrm{R})$ (Fig. 1). Population increases of introduced bacteria

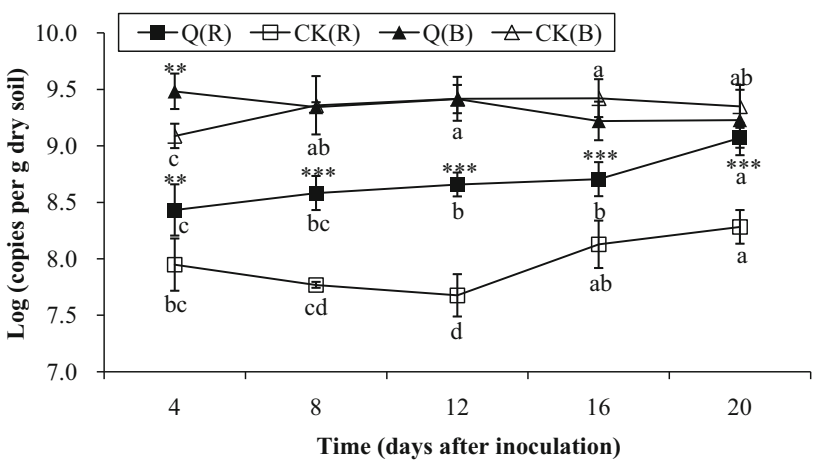

Fig. 1 Persistence of Gluconacetobacter liquefaciens qzr14 in the bulk (B) and rhizosphere (R) soil of cucumbers monitored during 20 days by real-time PCR (Asterisks indicate differences between Gluconacetobacter liquefaciens qzr14 inoculation (Q) and control check $(\mathrm{CK}), * * * P<0.001$; $* * P<0.01$; $* P<0.05$; Means in the line followed by the same letter are not significantly different at a $5 \%$ level of significance)

in rhizosphere soil were only observed in some rhizobia (Robert and Schmidt 1983; Steinberg et al. 1989). There might be three reasons for the successful colonization and persistence of G. liquefaciens qzr14 in the rhizosphere soil. First, the number of G. liquefaciens increased by fourfold in $\mathrm{Q}(\mathrm{R})$ but only twofold in $\mathrm{CK}(\mathrm{R})$ after inoculation, indicating that the introduced strain qzr14 can reproduce in rhizosphere soil. Second, strain qzr14 is an indigenous microorganism, since it can be detected in CK. It might have the advantage of adapting to the abiotic and biotic factors in the rhizosphere soil environment (vanVeen et al. 1997). Third, the increase in the number of strain qzr14 whether in $\mathrm{Q}$ or in $\mathrm{CK}$ may be related to the enhancement

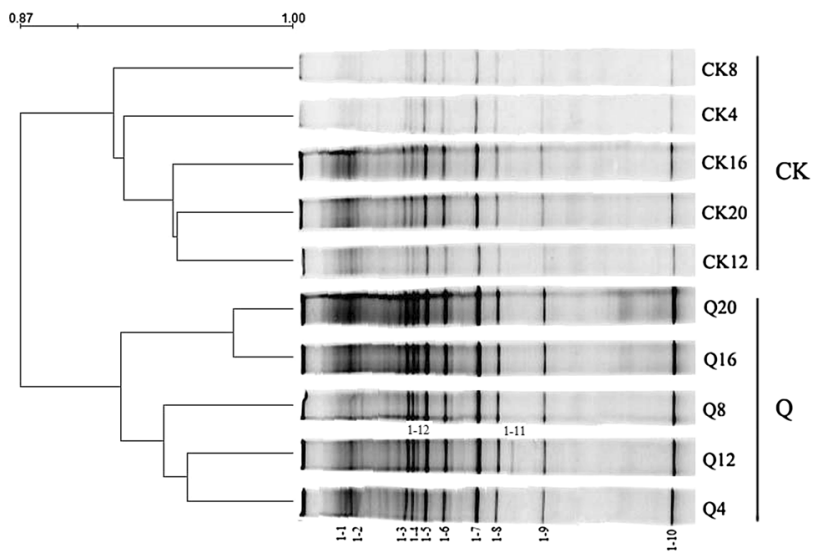

Fig. 2 Cluster analysis of denaturing gradient gel electrophoresis (DGGE) results of 16S rDNA amplicons from the rhizosphere soil of cucumber using UPMGA. (Bands marked with letters were excised from the polyacrylamide gel and sequenced. Q, Gluconacetobacter liquefaciens qzr14 inoculation; CK, control checks; Q4, Q8, Q12, Q16, and Q20 represent 4, 8, 12, 16, and 20 days after G. liquefaciens qzr14 inoculation; C4, C8, C12, C16, and C20, represent corresponding five control checks) 
Table 1 Effect of Gluconacetobacter liquefaciens qzr14 inoculation on bacterial diversity

\begin{tabular}{|c|c|c|c|c|c|c|c|c|c|c|}
\hline \multirow[t]{2}{*}{ Shannon-Weaver index } & \multirow[t]{2}{*}{ Treatment } & \multicolumn{5}{|c|}{ Days after inoculation } & \multicolumn{2}{|c|}{ Treatment } & \multicolumn{2}{|l|}{ Time } \\
\hline & & 4 & 8 & 12 & 16 & 20 & $t$ & $P$ & $F$ & $P$ \\
\hline \multirow[t]{2}{*}{ Rhizosphere soil } & CK & 2.14 & 2.15 & 2.14 & 2.13 & 2.12 & -8.521 & 0.000 & 0.056 & 0.992 \\
\hline & Q & 2.24 & 2.24 & 2.32 & 2.27 & 2.27 & & & & \\
\hline \multirow[t]{2}{*}{ Bulk soil } & CK & 2.08 & 2.06 & 2.03 & 2.23 & 2.27 & -3.664 & 0.021 & 0.277 & 0.881 \\
\hline & Q & 2.31 & 2.31 & 2.32 & 2.30 & 2.32 & & & & \\
\hline
\end{tabular}

Bold values indicate statistically significant

$Q$ Gluconacetobacter liquefaciens qzr14 inoculation, $C K$ control checks

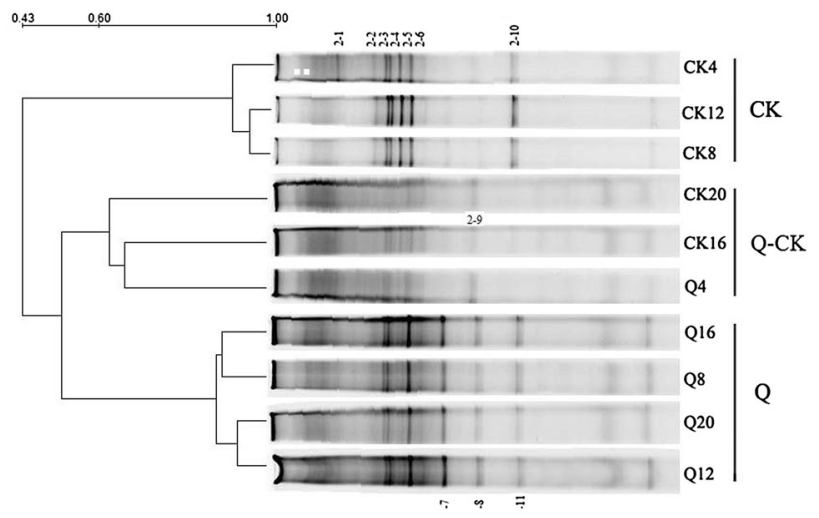

Fig. 3 Cluster analysis of denaturing gradient gel electrophoresis (DGGE) results of $16 \mathrm{~S}$ rDNA amplicons from the bulk soil of cucumber using UPMGA. (Bands marked with letters were excised from the polyacrylamide gel and sequenced. Q, Gluconacetobacter liquefaciens qzr14 inoculation; CK, control checks; Q4, Q8, Q12, $\mathrm{Q} 16$, and Q20 represent $4,8,12,16$, and 20 days after $G$. liquefaciens qzr14 inoculation; $\mathrm{C} 4, \mathrm{C} 8, \mathrm{C} 12, \mathrm{C} 16$, and $\mathrm{C} 20$, represent corresponding five control checks)

in plant secretions with cucumber growth as these can supply enough nutrients to soil microorganisms (Vacheron et al. 2013).

Studies on the colonization and persistence of introduced PGPM in bulk soil were limited. Some have shown that the inoculants' number is significantly higher in the rhizosphere than that in unplanted soil (Bashan et al. 1995; Steinberg et al. 1989). Our results showed that the number of $G$. liquefaciens in bulk soil was significantly higher than that in rhizosphere soil of cucumber except for $Q(R)$ and $\mathrm{Q}(\mathrm{B})$ at the 20th day after inoculation $(P<0.01)$ (Fig. 1). The lower number of $G$. liquefaciens in the rhizosphere soil might due to the reassembly of microbial communities in the rhizosphere as the original microbial community had been disrupted during transplant.

In bulk soil, the number of $G$. liquefaciens was significantly higher in inoculation treatment $[\mathrm{Q}(\mathrm{B})]$ than that in control check $[\mathrm{CK}(\mathrm{B})](P<0.01)$ only at the 4 th day after inoculation. Time had minimal effects on the number of G. liquefaciens in $\mathrm{Q}(\mathrm{B})$ and $\mathrm{CK}(\mathrm{B})$ (Fig. 1). This indicated that the number of $G$. liquefaciens did not differ between $\mathrm{Q}$ and $\mathrm{CK}$ in the bulk soil, and the number of $G$. liquefaciens in the bulk soil did not change with cucumber growth, which might due to soil capacity. It had been suggested that each soil ecosystem had its own distinctive biological space with respect to the maximum level of microbial biomass (King and Parke 1996).

\section{The effects of $G$. liquefaciens qzr14 inoculation on soil bacterial community}

The clustering of DGGE banding patterns of the rhizosphere soil showed a clear separation, with two main groups, one formed by $\mathrm{Q}$ and another by CK (Fig. 2). Q had higher bacterial diversity $(P<0.001)$ than CK in rhizosphere soil (Table 1). This indicated that $G$. liquefaciens qzr14 inoculation had a significant effect on bacterial community of rhizosphere soil. Twelve bacterial bands were chosen for further analysis. Bands 1-2, 1-3, 1-4, 1-5, $1-6,1-7,1-8,1-9,1-10$, and 1-12 were present in all treatments; However, these bands showed a four-fold higher intensity in Q (70-250) than that in CK (0-220). The clustering of DGGE banding patterns of bulk soil resulted in three main groups (Fig. 3). CK4, CK8, and CK12 constituted one main cluster (CK), Q8, Q12, Q16, and Q20 comprised the second main cluster $(\mathrm{Q})$, and Q4, CK16, and CK20 made up the third cluster (Q-CK). Q had higher bacterial diversity $(P<0.001)$ than $\mathrm{CK}$ in bulk soil (Table 1). This indicated that $G$. liquefaciens qzr14 inoculation had some effects on bacterial community of bulk soil. A total of 11 bands were detected. Bands 2-2 (Acidithiobacillus) and 2-10 (Alicyclobacillus) were only detected in the CK cluster. Bands 2-7 (Mesorhizobium), 2-8 (Gluconacetobacter), and 2-11 (Uncultured Firmicutes) were only present in the Q cluster (Fig. 3). Effects on plant growth are not necessarily resulting from a direct effect of the inoculants and may be related to induction or repression of resident microbial populations. Some works showed that inoculants had no effect or a transient effect on microbial community; however, others evidenced a longterm effect. For example, Sinorhizobium spp. inoculation 
Fig. 4 Neighbor-joining phylogenetic tree of bacteria from bulk (blue rectangle) and rhizosphere (red triangle) soil of cucumber, based on the $16 \mathrm{~S}$ rRNA gene sequences of DGGE fragments. Values shown in each node of the phylogenetic tree are bootstrap values; 1000 bootstrap replicates were performed

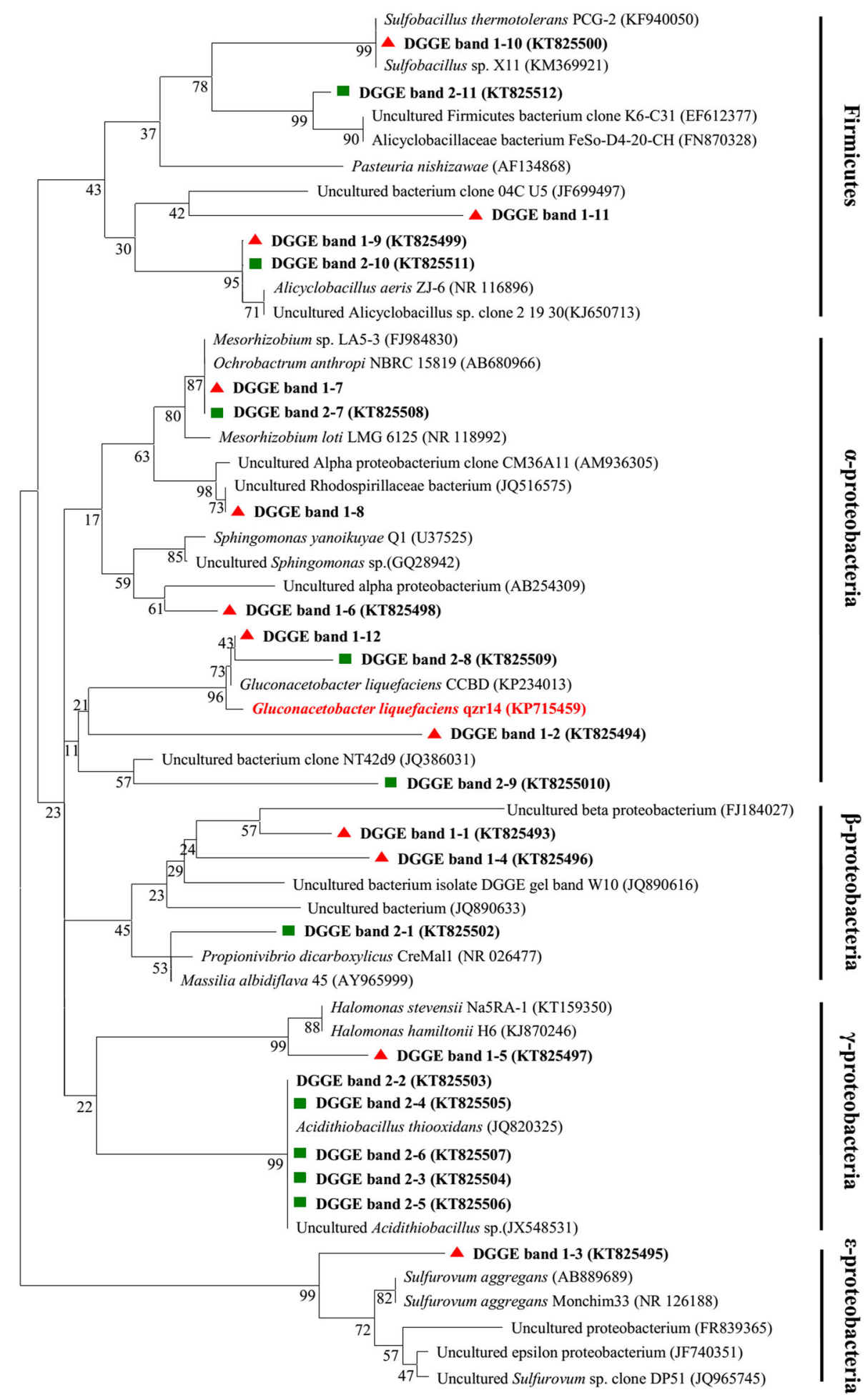

significantly affected bacterial communities in the rhizosphere of alfalfa (Babic et al. 2008; Schwieger and Tebbe 2000). Some rhizosphere microbiome inoculations could modify bacterial communities and change plant flowering time (Panke-Buisse et al. 2015). R. gallicum 8a3 and E. meliloti $4 \mathrm{H} 41$ inoculation showed significant effects on bacterial structure and diversity in the bulk soil of common bean (Trabelsi and Mhamdi 2013). In this study, we investigated the effect of G. liquefaciens qzr14 inoculation on bacterial community in rhizosphere and bulk soil simultaneously. This will further understood the promote mechanisms of inoculants on plant. 
A

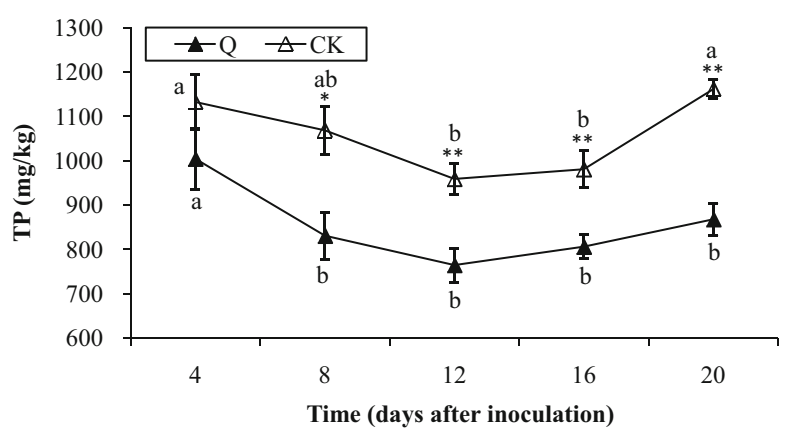

B

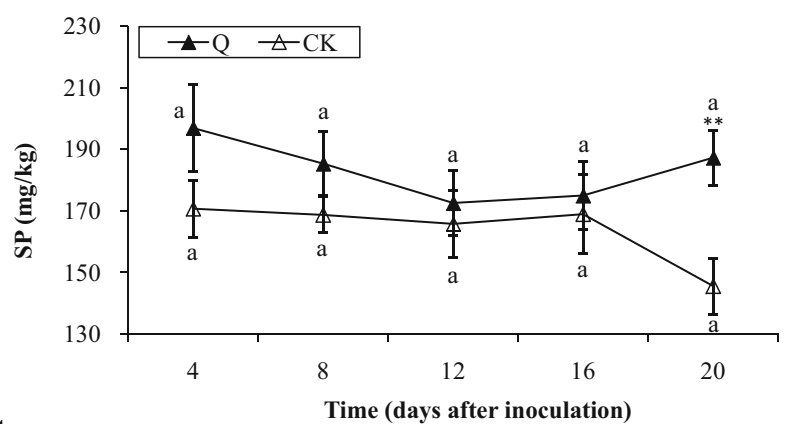

C

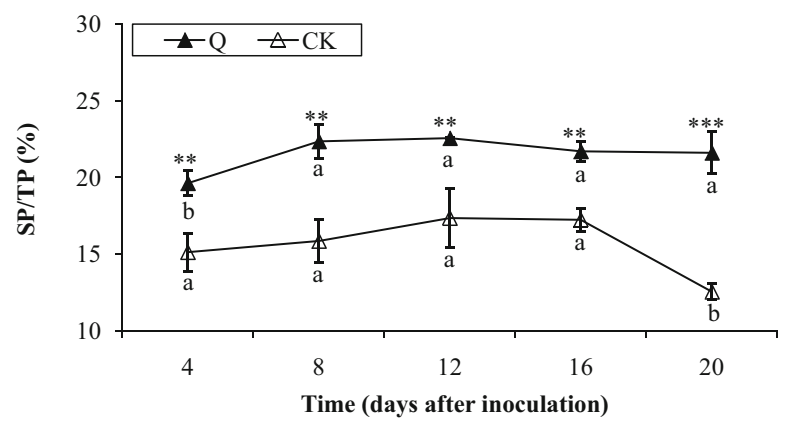

Fig. 5 Effects of Gluconacetobacter liquefaciens qzr14 inoculation on soil total phosphate (TP), soluble phosphate (SP), and the ratio of soluble phosphate to total phosphate (SP/TP) during 20 days after inoculation. (Asterisks indicate differences between G. liquefaciens qzr14 inoculation (Q) and control check (CK), ***P $<0.001$; $* * P<0.01 ; * P<0.05$; Means in the line followed by the same letter are not significantly different at a $5 \%$ level of significance)
Phylogenetic analysis of bacterial genes grouped all sequences into five clusters, namely, alpha, beta, gamma, epsilon Proteobacteria, and Firmicutes (Fig. 4). Epsilon proteobacteria were only present in rhizosphere soil. Gamma proteobacteria appeared in both rhizosphere and bulk soil. However, the only band in the rhizosphere belonged to genus Halomonas and all four bands in the bulk soil belonged to genus Acidithiobacillus. Beta proteobacteria also appeared in both rhizosphere and bulk soil. However, all two bands in the rhizosphere belonged to uncultured beta proteobacteria. The only band in the bulk soil was identified as genus Propionivibrivo. Alpha proteobacteria, especially G. liquefaciens, were shared by both rhizosphere and bulk soil except for two bands (identified with uncultured alpha proteobacteria) in the rhizosphere. Genus Sulfobacillus and one uncultured bacteria of Firmicutes were only observed in the rhizosphere soil. Another uncultured bacteria of Firmicutes were only identified in bulk soil. The difference in bacterial community composition of the rhizosphere and bulk soil might be related to variations in functions.

\section{The effects of $G$. liquefaciens qzr14 inoculation on soil phosphorus}

TP was higher in CK (958.93-1132.53\%) than that in Q (764.67-1004.40 $\mathrm{mg} \mathrm{kg}^{-1}$ ) during a period of 20 days after inoculation. TP was significantly lower at the 12th and 16th days after inoculation than that at the 4th and 20th days after inoculation in CK. TP was significantly higher at the 4th day after inoculation than the others in Q (Fig. 5a). SP was higher in Q (172.56-196.84 $\left.\mathrm{mg} \mathrm{kg}^{-1}\right)$ than in CK (145.63-170.73\%) during a period of 20 days after inoculation. Time had no significant effect on SP in both Q and CK (Fig. 5b). SP/TP was significantly higher in Q (19.62-22.57\%) than that in CK (12.53-17.35\%) during a period of 20 days after inoculation. The SP/TP was lowest at the 4th day after inoculation in $\mathrm{Q}$ and then significantly

Table 2 Effects of Gluconacetobacter liquefaciens qzr14 inoculation on the fresh weight of cucumber seedlings during 20 days

\begin{tabular}{llll}
\hline Days after inoculation & Q $(\mathrm{g})$ & CK $(\mathrm{g})$ & Rate of increase $(\%)$ \\
\hline 4 & $0.22 \pm 0.04 \mathrm{~d}$ & $0.13 \pm 0.02 \mathrm{~d}$ & 69.23 \\
8 & $0.47 \pm 0.09 \mathrm{c}$ & $0.41 \pm 0.06 \mathrm{c}$ & 14.63 \\
12 & $0.71 \pm 0.08 \mathrm{~b}^{*}$ & $0.51 \pm 0.02 \mathrm{c}$ & 39.22 \\
16 & $0.87 \pm 0.16 \mathrm{~b}$ & $0.76 \pm 0.04 \mathrm{~b}$ & 14.47 \\
20 & $1.15 \pm 0.04 \mathrm{a}$ & $1.05 \pm 0.18 \mathrm{a}$ & 9.52 \\
\hline
\end{tabular}

Values with different letters represent significant differences

Asterisks indicate differences between Gluconacetobacter liquefaciens qzr14 inoculation (Q) and control check $(\mathrm{CK}), * P<0.05$ 
Table 3 Correlations of fresh weight of cucumber seedlings, Gluconacetobacter liquefaciens population, bacterial diversity, and soil phosphorus

\begin{tabular}{|c|c|c|c|c|c|c|c|c|}
\hline & FW & NR & NB & DR & DB & $\mathrm{TP}$ & SP & SP/TP \\
\hline FW & 1 & & & & & & & \\
\hline NR & 0.563 & 1 & & & & & & \\
\hline NB & -0.041 & -0.144 & 1 & & & & & \\
\hline DR & 0.204 & $0.813 * *$ & 0.022 & 1 & & & & \\
\hline DB & 0.494 & $0.905 * *$ & 0.150 & 0.701* & 1 & & & \\
\hline TP & -0.286 & $-0.644^{*}$ & -0.121 & $-0.849 * *$ & -0.541 & 1 & & \\
\hline SP & -0.303 & 0.476 & 0.065 & 0.625 & 0.384 & -0.488 & 1 & \\
\hline SP/TP & 0.130 & 0.712* & 0.081 & $0.912 * *$ & 0.598 & $-0.950 * *$ & 0.724* & 1 \\
\hline
\end{tabular}

Bold values indicate statistically significant

$F W$ fresh weight of cucumbers seedlings, $N R$ log copy numbers of Gluconacetobacter liquefaciens qzr14 per g cucumber rhizosphere soil, $N B$ $\log$ copies numbers of $G$. liquefaciens qzr14 per g cucumber bulk soil, $D R$ the bacterial diversity of cucumber rhizosphere soil, $D B$ bacterial diversity of cucumber bulk soil, $T P$ total phosphate, $S P$ solubilized phosphate

** $P<0.01 ; * P<0.05$

Fig. 6 Performance of Gluconacetobacter liquefaciens qzr14 in the rhizosphere and bulk soil of cucumber

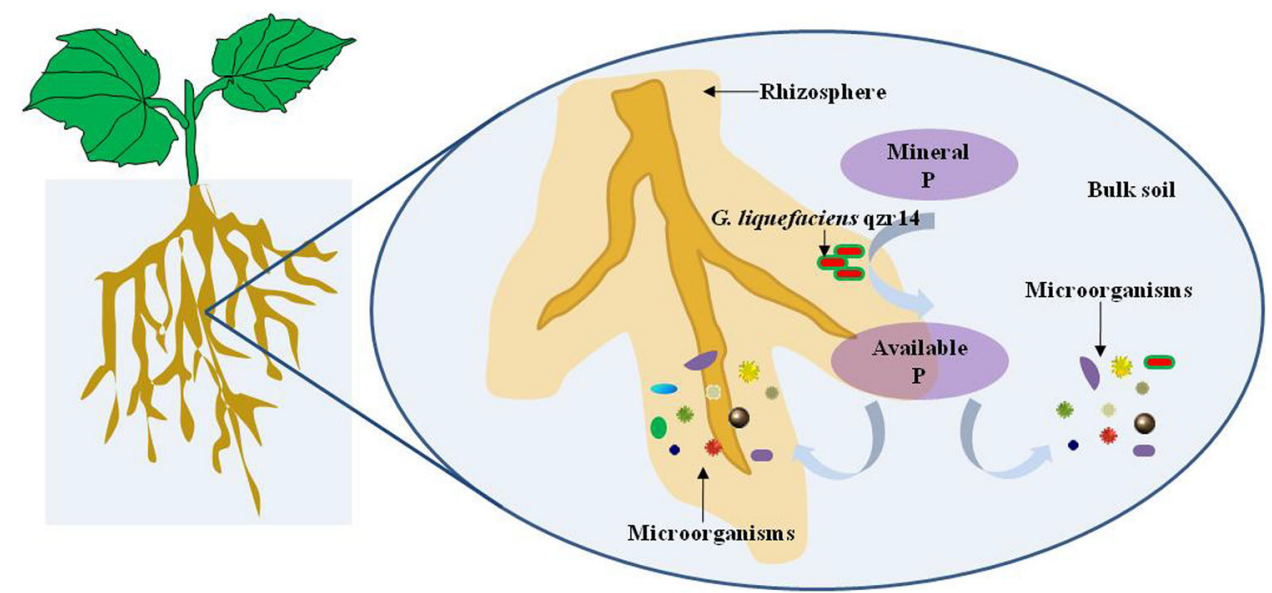

increased at the 8th day after inoculation. The SP/TP in CK dramatically decreased at the 20th day after inoculation (Fig. 5c). After a successful colonization, PGPMs would exert its ability in soil. Our results showed that the introduced G. liquefaciens qzr14 can exert its P-solubilizing ability in soil, as soil TP significantly decreased and SP/TP significantly increased after $G$. liquefaciens qzr14 inoculation. SP/TP was an effective index for the evaluation of phosphorus solubilizing ability of microorganisms. TP and SP are usually influenced by plants. The absorption of available phosphorus by inoculated plant in soil was more than that by non-inoculated plant as inoculated plant growth was faster than that of non-inoculated plants.

\section{The effects of G. liquefaciens qzr14 inoculation on cucumber seedlings}

The fresh weight of cucumber seedlings with G. liquefaciens qzr14 inoculation (Q) was higher than that of CK during a period of 20 days after inoculation. G. liquefaciens qzr14 inoculation (Q) increased cucumber seedling fresh weight from 9.52 to $69.23 \%$ (Table 2). After 12 days inoculation, the fresh weight of cucumber seedlings in $\mathrm{Q}$ was significantly higher than that in CK $(P<0.05)$ (Table 2). The fresh weights of cucumber seedling significantly increased over time in both qzr14 $(P<0.01)$ and CK $(P<0.01)($ Table 2$)$.

\section{Correlations on the number of G. liquefaciens, bacterial diversity, soil phosphorus, and the fresh weight of cucumber seedlings}

The number of $G$. liquefaciens in the rhizosphere soil was significantly positively related to bacterial diversity of rhizosphere $(r=0.813, P<0.01)$ and bulk $(r=0.905$, $P<0.01)$ soil and SP/TP $(r=0.712, P<0.05)$ and was significantly negatively related to TP $(r=-0.644$, $P<0.05$ ) (Table 3). The bacterial diversity in the 
rhizosphere soil was significantly positively related to bacterial diversity in the bulk soil $(r=0.701, P<0.05)$ and SP/TP $(r=0.912, P<0.01)$ and was significantly negatively related to TP $(r=0.849, P<0.01)$. TP was negatively related to SP/TP $(P<0.01)$. SP was positively related to SP/TP $(P<0.05)$. The fresh weight of cucumber seedlings and the number of qzr14 in cucumber bulk soil had no significant correlation with other parameters (Table 3). The use of phosphate-solubilizing bacteria as inoculants always increases $\mathrm{P}$ uptake by the plant and crop yield (Rodríguez and Fraga 1999). However, G. liquefaciens qzr14 did not promote cucumber growth by solubilizing phosphate, because the fresh weight of cucumber seedlings had a lower correlative with phosphorus and higher correlative with G. liquefaciens number in the rhizosphere soil. G. liquefaciens qzr14 inoculation had significant positive effects on bacterial diversity and biomass. Several reports indicated that the application of microbial inoculants can influence, at least temporarily, the resident microbial community's composition and function by directly inducing trophic competitions and antagonistic/ synergic interactions with resident microbial populations or indirectly mediating via enhanced root growth and exudation (Trabelsi and Mhamdi 2013). It was inferred that the introduced G. liquefaciens qzr14 effectively colonized the rhizosphere soil of cucumber and then expanded the rhizosphere bacterial communities by solubilizing soil phosphorus and supplying soluble phosphorus (Fig. 6). The expanded bacterial communities might promote cucumber growth by some new functions (Saikaly and Oerther 2011).

\section{Conclusion}

In summary, we found that the introduced Gluconacetobacter liquefaciens qzr14 effectively colonized in rhizosphere other than bulk soil of cucumber. The effectively colonized G. liquefaciens qzr14 might expand rhizosphere bacterial community by solubilizing soil phosphorus. The expanded bacterial communities might promote cucumber growth by some new functions.

\begin{abstract}
Acknowledgements This study was supported by the National Natural Science Foundation of China (no. 31500424), Science and Technology Service Network Initiative (no. KFJ-SW-STS-143-6), Tianjin Science and Technology Plan Project (16YDYGHZ00060), Tianjin Science and Technology Plan Project (15ZCZDSF00620), and Open Fund of Zhejiang Provincial Top Key Discipline of Aquaculture in Ningbo University (Grant no. xkzsc10).
\end{abstract}

\section{Compliance with ethical standards}

Conflict of interest All the authors declare that they have no financial/commercial conflicts of interest.

\section{References}

Babic KH, Schauss K, Hai B, Sikora S, Redzepovic S, Radl V, Schloter M (2008) Influence of different Sinorhizobium meliloti inocula on abundance of genes involved in nitrogen transformations in the rhizosphere of alfalfa (Medicago sativa L.). Environ Microbiol 10:2922-2930. doi:10.1111/j.1462-2920.2008.01762.x

Baris O, Sahin F, Turan M, Orhan F, Gulluce M (2014) Use of plantgrowth-promoting rhizobacteria (pgpr) seed inoculation as alternative fertilizer inputs in wheat and barley production. Commun Soil Sci Plant Anal 45:2457-2467

Bashan Y, Puente ME, Rodriguezmendoza MN, Toledo G, Holguin G, Ferreracerrato R, Pedrin S (1995) Survival of Azospirillum brasilense in the bulk soil and rhizosphere of 23 soil types. Appl Environ Microbiol 61:1938-1945

Bernabeu PR, Pistorio M, Torres-Tejerizo G, Estrada-De los Santos P, Galar ML, Boiardi JL, Luna MF (2015) Colonization and plant growth-promotion of tomato by Burkholderia tropica. Sci Hortic (Amsterdam) 191:113-120. doi:10.1016/j.scienta.2015.05.014

Bhattacharjee RB, Jourand P, Chaintreuil C, Dreyfus B, Singh A, Mukhopadhyay SN (2012) Indole acetic acid and ACC deaminase-producing Rhizobium leguminosarum bv. trifolii SN10 promote rice growth, and in the process undergo colonization and chemotaxis. Biol Fertil Soils 48:173-182

Bhattacharyya PN, Jha DK (2012) Plant growth-promoting rhizobacteria (PGPR): emergence in agriculture. World J Microbiol Biotechnol 28:1327-1350. doi:10.1007/s11274-011-0979-9

Bloemberg GV, Lugtenberg BJ (2001) Molecular basis of plant growth promotion and biocontrol by rhizobacteria. Curr Opin Plant Biol 4:343-350

Chabot R, Antoun H, Kloepper JW, Beauchamp CJ (1996) Root colonization of maize and lettuce by bioluminescent Rhizobium leguminosarum biovar phaseoli. Appl Environ Microbiol 62:2767-2772

Chen Y, Rekha P, Arun A, Shen F, Lai W-A, Young C (2006) Phosphate solubilizing bacteria from subtropical soil and their tricalcium phosphate solubilizing abilities. Appl Soil Ecol 34:33-41

Compant S, Clement C, Sessitsch A (2010) Plant growth-promoting bacteria in the rhizo- and endosphere of plants: their role, colonization, mechanisms involved and prospects for utilization. Soil Biol Biochem 42:669-678. doi:10.1016/j.soilbio.2009.11. 024

Couillerot O, Bouffaud M-L, Baudoin E, Muller D, CaballeroMellado J, Moënne-Loccoz Y (2010) Development of a realtime PCR method to quantify the PGPR strain Azospirillum lipoferum CRT1 on maize seedlings. Soil Biol Biochem 42:2298-2305

Dudeja SS, Khurana AL (1989) Persistence of Bradyrhizobium sp. (Cajanus) in a sandy loam. Soil Biol Biochem 21:709-713. doi:10.1016/0038-0717(89)90068-0

Franke IH, Fegan M, Hayward C, Leonard G, Stackebrandt E, Sly LI (1999) Description of Gluconacetobacter sacchari sp nov., a new species of acetic acid bacterium isolated from the leaf sheath of sugar cane and from the pink sugar-cane mealy bug. Int J Syst Bacteriol 49:1681-1693

Franke-Whittle IH, O’Shea MG, Leonard GJ, Sly LI (2005) Design, development, and use of molecular primers and probes for the detection of Gluconacetobacter species in the pink sugarcane mealybug. Microb Ecol 50:128-139. doi:10.1007/s00248-0040138-z

Holmberg AIJ, Melin P, Levenfors JP, Sundh I (2012) Fate and behaviour of a seed-applied Pseudomonas brassicacearum strain in a winter wheat field trial, as determined by analysis with 
SCAR markers. Biocontrol Sci Technol 22:379-392. doi:10. 1080/09583157.2012.661404

Jiménez DJ, Montaña JS, Martínez MM (2011) Characterization of free nitrogen fixing bacteria of the genus Azotobacter in organic vegetable-grown Colombian soils. Braz J Microbiol 42:846-858

King EB, Parke JL (1996) Population density of the biocontrol agent Burkholderia cepacia AMMDR1 on four pea cultivars. Soil Biol Biochem 28:307-312. doi:10.1016/0038-0717(95)00147-6

Kumar S, Pandey P, Maheshwari D (2009) Reduction in dose of chemical fertilizers and growth enhancement of sesame (Sesamum indicum L.) with application of rhizospheric competent Pseudomonas aeruginosa LES4. Eur J Soil Biol 45:334-340

Liu W, Yang C, Shi S, Shu W (2014) Effects of plant growthpromoting bacteria isolated from copper tailings on plants in sterilized and non-sterilized tailings. Chemosphere 97:47-53

Luna MF, Aprea J, Crespo JM, Boiardi JL (2012) Colonization and yield promotion of tomato by Gluconacetobacter diazotrophicus. Appl Soil Ecol 61:225-229. doi:10.1016/j.apsoil.2011.09.002

Munoz-Rojas J, Caballero-Mellado J (2003) Population dynamics of Gluconacetobacter diazotrophicus in sugarcane cultivars and its effect on plant growth. Microb Ecol 46:454-464. doi:10.1007/ s00248-003-0110-3

Nautiyal CS (1999) An efficient microbiological growth medium for screening phosphate solubilizing microorganisms. FEMS Microbiol Lett 170:265-270. doi:10.1016/S0378-1097(98)00555-2

Owen D, Williams A, Griffith G, Withers P (2015) Use of commercial bio-inoculants to increase agricultural production through improved phosphrous acquisition. Appl Soil Ecol 86:41-54

Panke-Buisse K, Poole AC, Goodrich JK, Ley RE, Kao-Kniffin J (2015) Selection on soil microbiomes reveals reproducible impacts on plant function. ISME J 9:980-989. doi:10.1038/ ismej.2014.196

Pellegrino E, Turrini A, Gamper HA, Cafà G, Bonari E, Young JPW, Giovannetti M (2012) Establishment, persistence and effectiveness of arbuscular mycorrhizal fungal inoculants in the field revealed using molecular genetic tracing and measurement of yield components. New Phytol 194:810-822

Postma J, Hok-A-Hin CH, Oude Voshaar JH (1990) Influence of the inoculum density on the growth and survival of Rhizobium leguminosarum biovar trifolii introduced into sterile and nonsterile loamy sand and silt loam. FEMS Microbiol Lett 73:49-57. doi:10.1016/0378-1097(90)90723-4

Rana A, Saharan B, Joshi M, Prasanna R, Kumar K, Nain L (2011) Identification of multi-trait PGPR isolates and evaluating their potential as inoculants for wheat. Ann Microbiol 61:893-900

Robert FM, Schmidt EL (1983) Population changes and persistence of Rhizobium phaseoli in soil and rhizospheres. Appl Environ Microbiol 45:550-556

Rodríguez H, Fraga R (1999) Phosphate solubilizing bacteria and their role in plant growth promotion. Biotechnol Adv 17:319-339

Saikaly P, Oerther D (2011) Diversity of dominant bacterial taxa in activated sludge promotes functional resistance following toxic shock loading. Microb Ecol 61:557-567. doi:10.1007/s00248010-9783-6

Saravanan VS, Madhaiyan M, Osborne J, Thangaraju M, Sa TM (2008) Ecological occurrence of Gluconacetobacter diazotrophicus and nitrogen-fixing Acetobacteraceae members: their possible role in plant growth promotion. Microb Ecol 55:130-140. doi:10.1007/s00248-007-9258-6

Sathya A, Vijayabharathi R, Srinivas V, Gopalakrishnan S (2016) Plant growth-promoting actinobacteria on chickpea seed mineral density: an upcoming complementary tool for sustainable biofortification strategy. 3 Biotech 6:1-6. doi:10.1007/s13205016-0458-y

Schwieger F, Tebbe CC (2000) Effect of field inoculation with Sinorhizobium meliloti L33 on the composition of bacterial communities in rhizospheres of a target plant (Medicago sativa) and a non-target plant (Chenopodium album) - linking of $16 \mathrm{~S}$ rRNA gene-based single-strand conformation polymorphism community profiles to the diversity of cultivated bacteria. Appl Environ Microbiol 66:3556-3565. doi:10.1128/Aem.66.8.35563565.2000

Steinberg C, Gamard P, Faurie G, Lensi R (1989) Survival and potential denitrifying activity of Azospirillum lipoferum and Bradyrhizobium japonicum inoculated into sterilized soil. Biol Fertil Soils 7:101-107. doi:10.1007/Bf00292566

Tanner MA, Shoskes D, Shahed A, Pace NR (1999) Prevalence of corynebacterial 16S rRNA sequences in patients with bacterial and "nonbacterial" prostatitis. J Clin Microbiol 37:1863-1870

Trabelsi D, Mhamdi R (2013) Microbial inoculants and their impact on soil microbial communities: a review. Biomed Res Int. doi: $10.1155 / 2013 / 863240$

Troxler J, Svercel M, Natsch A, Zala M, Keel C, Moënne-Loccoz Y, Défago G (2012) Persistence of a biocontrol Pseudomonas inoculant as high populations of culturable and non-culturable cells in 200-cm-deep soil profiles. Soil Biol Biochem 44:122-129

Vacheron $J$ et al (2013) Plant growth-promoting rhizobacteria and root system functioning. Front Plant Sci 4:356. doi:10.3389/fpls. 2013.00356

Vanelsas JD, Dijkstra AF, Govaert JM, Vanveen JA (1986) Survival of Pseudomonas fluorescens and Bacillus subtilis introduced into 2 soils of different texture in field microplots. FEMS Microbiol Ecol 38:151-160. doi:10.1016/0378-1097(86)90046-7

vanVeen JA, vanOverbeek LS, vanElsas JD (1997) Fate and activity of microorganisms introduced into soil. Microbiol Mol Biol Rev 61:121-135

Von Felten A, Defago G, Maurhofer M (2010) Quantification of Pseudomonas fluorescens strains F113, CHA0 and Pf153 in the rhizosphere of maize by strain-specific real-time PCR unaffected by the variability of DNA extraction efficiency. J Microbiol Methods 81:108-115. doi:10.1016/j.mimet.2010.02.003

Wang J, Zhang H, Li X, Su Z, Li X, Xu M (2014) Effects of tillage and residue incorporation on composition and abundance of microbial communities of a fluvo-aquic soil. Eur J Soil Biol 65:70-78. doi:10.1016/j.ejsobi.2014.10.003

Xie CS, Xu J, Tang J, Baig SA, Xu XH (2013) Comparison of phosphorus determination methods by ion chromatography and molybdenum blue methods. Commun Soil Sci Plan 44:2535-2545. doi:10.1080/00103624.2013.811518

Yin T et al (2015) The screening of efficient phosphorus-solubilizing bacteria and the primary study on its mechanism of plantgrowth-promoting. Biotechnol Bull 31:234-242 (in Chinese) 